\title{
Telemonitoring Devices and Systems: Current Status and Future Trends
}

\author{
Liliana Tavares Machadeiro, Pedro Dinis Gaspar, and Miguel Castelo-Branco
}

\section{Introduction}

The number of individuals suffering from one of the various chronic diseases, which according to the World Health Organization (WHO) may also be referred to as no communicable diseases, has been increasing [1]. The significant increase in the average life expectancy at birth in recent years is an important and contributory factor, resulting in a greater focus on the disease in the elderly, who in future will be the large part of the population. These diseases are largely responsible for the expense of national health systems, which nowadays face major problems with scarce financial and human resources. From the point of view of the chronic patient, the expenses with the disease are high due to the medication costs and continuous monitoring and support that the disease demands [2]. Chronic patients are considered the main responsible for the increasing use of medical, hospital and emergency services. Thus, a special care that considers monitoring certain physical and biological parameters associated with the disease is required [3].

So, the need to find solutions to provide healthcare where good management of chronic diseases is performed, addressing the problem of the shortage of health

\author{
L. T. Machadeiro \\ University of Beira Interior, Covilhã, Portugal \\ P. D. Gaspar $(\varangle)$ \\ University of Beira Interior, Covilhã, Portugal \\ C-MAST - Centre for Mechanical and Aerospace Science and Technologies, \\ University of Beira Interior, Covilhã, Portugal \\ e-mail: dinis@ubi.pt \\ M. Castelo-Branco \\ University of Beira Interior, Covilhã, Portugal \\ CICS-UBI - Health Sciences Research Centre, University of Beira Interior, Covilhã, Portugal


professionals by effectively allocating the available resources [4], is growing and it is believed that passes through telehealth devices and/or systems [5]. Telehealth, e-health, m-health and telemonitoring are quickly evolving and are already part of health systems. However, in the future, telemonitoring may become critical in the treatment of chronic diseases [3].

Telemonitoring consists in accomplishing the distant monitoring of patients, i.e., monitoring them remotely from the comfort of their home or beyond it [6]. Thus, there is an automonitoring of biological and physical parameters of the patient outside the hospital environment performed by itself or by a health provider, and the data is transmitted to the health care systems using information and communications technology (ICT).

The use of telemonitoring devices and systems can provide a safe and effective monitoring of these patients. The measurement of physical and biological parameters essential for the analysis of the evolution and treatment of the disease prescribed by the health professionals allows them to evaluate the data and take decisions to change or maintain prescriptions or health care for the monitored patient $[4,7]$.

Telemonitoring combines several advantages for patients with chronic diseases as well as for health systems. For the patient, telemonitoring allows the promotion of a better self-control that results in a better quality of life [2]. In the background, it allows the early detection of warning signs that consequently decrease the hospitalizations of these patients [4]. For the healthcare systems, the lack of health professionals and the lack of financial resources can be diminished by telemonitoring these patients. Health institutions will in future experience difficulties in caring for patients and their hospitalization. The growing number of patients is related also to the increasing trend of average life hope [4].

It is perceived that there is a problem in the health systems that will follow a worldwide worsening trend in the near future. The part of this problem can be surpassed with the growth of telemonitoring in the health sector, and particularly fostering the telemonitoring physical and/or biological parameters of patients with different chronic diseases.

This paper describes the evaluation carried out concerning the current status of telemonitoring devices and systems existing in the market and in some cases in the development phase. Their technical specifications, characteristics and their advantages for patients and health systems are described. In addition, the future trend for the development of this type of devices and system is analysed. Proposals for the implementation of new telemonitoring devices and systems for the support and management of chronic diseases are performed.

\section{Methods}

It is essential to introduce telemonitoring devices and systems into the daily life of chronically ill patients. As this condition is becoming a reality, a research and a description of some of market-available telemonitoring devices and systems 
applied to different chronic pathologies is performed. The selection of chronic diseases described within the paper was performed according to two criteria: (1) prevalence of chronic diseases, and preference was given to those with the highest incidence in the population; (2) frequency of information found in academic and scientific databases, such as PubMed, IEEE and B-on. Additionally, the technical specifications were gathered from the web pages of companies manufacturing or distributing telemonitoring devices and systems referenced in reports prepared by the Market Reports Center.

Proposals are performed to improve the technical specifications of the future telemonitoring devices and/or systems based on the ones existing in the market.

\section{State of the Art on IoT Telemonitoring Medical Devices}

The telemonitoring devices and systems discussed in this article were HealthGO Mini, VITALS360 ${ }^{\circledR}$, CareHomePod and HomePod, iSpO2 ${ }^{\circledR}$, EQ Connect ${ }^{\mathrm{TM}}$, eNephro, Prototype Device, eCareCompanion, Genesis Telemonitor, Honeywell HomMed, TCare Cardio Monitor and Smartheart ${ }^{\mathrm{TM}}$. These telemonitoring devices and systems are applied to chronic diseases such as Diabetes mellitus, Chronic Obstructive Pulmonary Disease (COPD) and Asthma, Chronic Kidney Disease, Thromboembolic Diseases and Heart Failure.

\subsection{Telemonitoring Devices Used in Diabetes Mellitus}

Diabetes mellitus is characterized as being a metabolic disease in which there is hyperglycaemia resulting from defective insulin secretion, insulin action or both. Chronic hyperglycaemia of diabetes is associated with long-term damage, such as dysfunction and failure of various organs, especially eyes, kidneys and heart [8]. According to the International Diabetes Federation (IDF) in 2011, 35 million adults had Type 1 or Type 2 diabetes. The estimate for 2030 is that there are 43 million adults with diabetes [9].

Diabetes management in Europe alone entails huge costs for health systems. In 2011, 89 million euros were spent on the treatment of diabetes and its complications, with no indirect costs being accounted for, which can increase the associated costs dramatically [9]. In the year 2013, IDF accounted for about 206 million diagnosed diabetics globally. The costs associated with Type 2 diabetic patients alone are about two to three times higher compared to a person of the same sex and the same age without the disease. Approximately $80 \%$ of the costs that this disease brings to health systems are the treatment of complications resulting from poor management of the disease [10]. 
According to IDF, the costs associated with diabetes will increase greatly due not only to the expected increase in the number of patients but also to the associated costs due to complications caused by poor management of the disease [9].

Monitoring glycaemic control in diabetic patients is essential. This procedure, repeated throughout the day along with taking the correct medication is essential to avoid complications of the disease. These patients have direct contact with the specialty doctor on average every 3 months or every 4 months, where adjustments are made to the therapy. In the intervals of these consultations, the dosages of medication may have to be changed to an efficacy of disease management. This is made difficult by the fact that the patient does not have the medical advice to do so [10].

Following is a set of commercially available diabetes mellitus telemonitoring devices. The devices described are HealthGO Mini, VITALS360 ${ }^{\circledR}$ and CareHomePod and HomePod. The CareHomePod is used in multi-resident homes and the HomePod is used in singular-resident homes. All devices have the portable advantage, allowing patients to take them in the event of a need to travel. The HealthGO Mini and CareHomePod and HomePod work as platforms that receive data collected by other devices, in this case, the blood glucose metre that diabetic patients use to obtain the blood glucose value that they connect with the platform (HealthGO Mini) via Bluetooth or USB and with the tablet (CareHomePod and HomePod) via Bluetooth. In the case of the VITALS $3600^{\circledR}$ device, it already has a built-in blood glucose metre that allows the blood glucose test to be performed directly on the device [11-13].

The process of obtaining the glycaemic value is invasive in all devices and platforms; being through the puncture to obtain a drop of blood that is placed on a test tape that is previously placed in the blood glucose metre. After this process, the glycaemic values obtained are sent through Wi-Fi to databases of each device that allow the analysis of these data by specialized health professionals who subsequently make the decision how to act. The device-to-patient interface is simple on all devices but customizable to the patient only VITALS360 ${ }^{\circledR}$ and CareHomePod and HomePod have this feature [11, 13, 14]. Table 1 compares the technical specifications of telemonitoring devices and systems found for Diabetes mellitus disease.

Table 1 Comparison of technical specifications of telemonitoring devices used in Diabetes mellitus

\begin{tabular}{l|l|l|l}
\hline & Device 1 & Device 2 & Device 3 \\
\hline Designation & HealthGO Mini & VITALS360 & CareHomePod; HomePod \\
\hline Portability & Yes & Yes & Yes \\
\hline Invasiveness & Yes & Yes & Yes \\
\hline Interface & Not customized and simple & Custom and simple & Custom and simple \\
\hline Connectivity & BluetoothUSBWi-Fi & Wi-Fi & BluetoothWi-Fi \\
\hline Interoperability & No & No & No \\
\hline
\end{tabular}


It is concluded that all the described devices present very similar technical specifications, being overall only distinguished by the functionalities related to the connectivity, which are fundamental for any telemonitoring device, as well as for the man-machine interface. Interoperability is the missing technical specification in the three telemonitoring devices and systems analysed. However, as long as there is no international regulation that mandates and imposes manufacturers to provide this functionality in their devices, all will continue to use closed proprietary systems. It is important to remember that interoperability between systems and devices will have to be followed by strict legal and regulatory policies and standards related to data encryption to ensure their confidentiality. Some steps are taken in this direction by the Food and Drug Administration (FDA) in the United States of America (USA) as well as the European Commission (EC) in the European Union (EU).

\subsection{Telemonitoring Devices Used in COPD and Asthma}

Chronic Obstructive Pulmonary Disease (COPD) is a very common disease and is characterized by a progressive decrease in lung capacity and acute respiratory exacerbations [15]. In COPD there is a limitation of progressive respiratory flow, which may be partially or totally irreversible, associated with a chronic inflammatory response that increases harmful particles or gases in the airways and lungs [16]. In this disease, the loss of respiratory muscle function and airway obstruction is progressive [16], resulting in frequent hospital admissions, incapacity for the patient with this disease and also depression [17].

These patients suffer with the gradual worsening of some symptoms and also of lung function for a few days, such as exacerbations, sinus tachycardia, dyspnoea and hypoxemia. The early recognition by patients of these serious symptoms is not always achieved in order to avoid hospitalization. These symptomatologic events contribute significantly to a worsening of the disease, by deteriorating the respiratory health of the patient, and consequently also contribute to a lower quality of life for the patient $[15,16]$.

It is estimated that by 2020 the costs associated with the disease in the USA reach $\$ 49$ billion annually. These costs are mostly due to hospital admissions due to exacerbations [18].

Asthma, which can be referred to as a form of COPD, is a disease that presents problems on a global scale and affects both the quality of life of patients who are carriers, as well as health systems because it is a high cost disease [19]. This disease is one of the most frequent chronic diseases and has been increasing its frequency in the last decade [20]. Only in the EU and the USA it affects about $5-6 \%$ of the population. Asthma and other forms of COPD require management, self-management and monitoring in order to control the symptoms of the disease with therapies adjusted to the condition of each patient and also to reduce the risk of exacerbations $[16,20]$. The vast majority of asthmatic patients undergo 
corticosteroid therapy by inhalation and with drugs called $\beta$-adrenergic agonists, and a proportion of these patients require additional drugs such as oral corticosteroids [20].

Some biological parameters are important both for the diagnosis of the disease, as well as for its monitoring in the hospital environment and daily in the home. Pulmonary function is a biological parameter that fits perfectly into this definition [19].

The devices analysed are CareHomePod and HomePod, VITALS $360^{\circledR}$ and iSpO $2^{\circledR}$. All devices have the portability characteristic, enabling patients to use them in the event of a need to travel. The $\mathrm{iSpO}^{\circledR}{ }^{\circledR}$ is an adaptive device for an android smartphone or iOS, which functions as a pulse oximeter [21]. In the case of CareHomePod and HomePod the oximeter sends the values collected for these devices through Bluetooth and the VITALS $360^{\circledR}$ has a built-in oximeter. Again, the processes of obtaining the values is not invasive in all devices and platforms. Then, measured values are sent through Wi-Fi to databases. The data is analysed by specialized health professionals who subsequently decide how to act. The interface of the device to the patient is simple on all devices and customizable to the patient $[12,21]$.

Table 2 reviews the technical specifications of the devices and telemonitoring systems found for COPD and Asthma.

The comparative results present the same conclusions as in the case of diabetes mellitus. Interoperability is the missing technical specification in the three devices and systems of telemonitoring analysed.

\subsection{Telemonitoring Systems Used in Chronic Kidney Disease}

Chronic kidney disease is a progressive disease that irreversibly impairs kidney structure and function [22].

Table 2 Comparison of technical specifications of telemonitoring devices used in DPOC and Asthma

\begin{tabular}{l|l|l|l}
\hline & Device 1 & Device 2 & Device 3 \\
\hline Designation & $\begin{array}{l}\text { CareHomePod; } \\
\text { HomePod }\end{array}$ & VITALS360 & iSpO2 $^{\circledR}$ \\
\hline Portability & Yes & Yes & Yes \\
\hline Invasiveness & No & No & No \\
\hline Interface & Custom and simple & Custom and simple & Custom and simple \\
\hline Connectivity & $\begin{array}{l}\text { Bluetooth } \\
\text { Wi-Fi }\end{array}$ & Wi-Fi & $\begin{array}{l}\text { Direct connection to } \\
\text { smartphone } \\
\text { Wi-Fi }\end{array}$ \\
\hline Interoperability & No & No & No \\
\hline & & &
\end{tabular}


Chronic kidney disease is associated with high morbidity, mortality and also high health expenditure. Factors such as diabetes mellitus, hypertension and cardiovascular diseases may contribute to the onset of chronic kidney disease. Thus, the prevalence of chronic kidney disease is increasing globally [23]. In the USA in 2006, the prevalence of chronic kidney disease in the adult population over 65 years of age at stage 3 was estimated to be 7.6 million patients at stage 4 of 400,000 patients and at stage 5 of 300,000 patients [24].

Chronic kidney patients in the early stages of the disease may be asymptomatic, leading to a lack of demand for medical care. Timely care of these patients can lead to a reduction in morbidity, mortality, costs associated with the disease, as well as a reduction in the number of consultations required for the patient, as the responsiveness of health systems tends to decrease $[25,26]$.

The economic burden on health systems increases as the disease progresses, with the greatest burden being on the last stage involving dialysis, hemodialysis or renal transplantation. Avoiding reaching these stages is important. Controlling the disease is fundamental, preventing its progression and consequently alleviating the economic burden, minimizing hospitalizations and improving the patient's quality of life [27].

In some situations not reaching the last stage of the disease becomes impossible. Peritoneal dialysis becomes the best solution for these patients. Telemonitoring combined with dialysis becomes a fundamental point, thus increasing the bet in this area [28].

In order to provide optimal care for these patients, telemonitoring becomes an essential component of health systems in the care of chronic kidney patients [25]. Telemonitoring combined with self-monitoring in these patients is essential in preventing disease progression and consequently in reducing hospitalizations [26].

Many telemonitoring systems applied to chronic kidney disease are still under development. In the following description of the devices, only the first device is already on the market. With all the rest only case studies were done to see if they are effective in the disease, having all had great success with proven effectiveness. The systems described are EQ Connect ${ }^{\mathrm{TM}}$, eNephro and Prototype Device. All systems present the portable advantage, enabling patients to use them aboard. This feature is largely due to the fact that they are web and android applications that receive the specific disease-related data predefined by specialized healthcare professionals through the manual introduction of data by the patient. The measuring process is non-invasive. The results are sent through $\mathrm{Wi}-\mathrm{Fi}$ to databases to be analysed by specialized health professionals. The device interface to the patient is simple and customizable to the patient in the EQ Connect ${ }^{\mathrm{TM}}$ and eNephro application. The Prototype Device (still in development) is not customizable to the patient [24, 26, 29].

Table 3 reviews the technical specifications of telemonitoring systems found for Chronic Kidney Disease. Again, the results are similar. Interoperability is the missing technical specification in the three telemonitoring systems analysed. 
Table 3 Comparison of technical specifications of telemonitoring systems used in Chronic Kidney Disease

\begin{tabular}{l|l|l|l}
\hline & Device 1 & Device 2 & Device 3 \\
\hline Designation & EQ Connect ${ }^{\mathrm{TM}}$ & eNephro & Prototype device \\
\hline Portability & Yes & Yes & Yes \\
\hline Invasiveness & No & No & No \\
\hline Interface & Custom and simple & Custom and simple & Not customized and simple \\
\hline Connectivity & Wi-Fi & Wi-Fi & Wi-Fi \\
\hline Interoperability & No & No & No \\
\hline
\end{tabular}

\subsection{Telemonitoring Devices Used in Thromboembolic Diseases}

Thrombosis is a pathology characterized by excess blood clotting that exists in vessel walls, thus becoming the desirable opposite of homeostasis, where there is coagulation necessary at specific sites of vessels where there is injury. This pathology occurs both in the arterial circulation and in the venous circulation and can trigger different pathologies depending on where it occurs, such as myocardial infarction, pulmonary embolism and ischemic stroke [30].

The possible thromboembolic events are prevented by therapeutic measures such as oral anticoagulants, such as warfarin, and the aim of this therapy is to preserve adjusted anticoagulation levels, preventing events with the lowest possible risk of haemorrhage [31, 32].

The devices described are eCareCompanion, Genesis Telemonitor and Honeywell HomMed. All systems are portable. Honeywell HomMed is the largest platform, thus less easy to transport. Obtaining the INR value is invasive and is performed by puncture to obtain a drop of blood that is placed on a test strip previously placed in an INR monitoring device. In eCareCompanion the value of INR is received via Bluetooth, in the case of Genesis Telemonitor the value is entered manually and in Honeywell HomMed receives the data through a cable connected to the INR monitoring device. Results are sent through Wi-Fi to databases in order to be analysed by specialized health professionals. The patient-to-patient interface is simple and customizable to the patient on all devices [33-35].

Table 4 reviews the technical specifications of devices and telemonitoring systems found for thromboembolic diseases. As in the previous cases, the interoperability between systems and devices is not available.

\subsection{Telemonitoring Devices Used in Heart Failure}

Heart failure is a disease that is growing in developed countries, despite the diminished incidence of cardiovascular diseases. It is estimated that there are 23 
Table 4 Comparison of technical specifications of telemonitoring systems used in Thromboembolic Diseases

\begin{tabular}{l|l|l|l}
\hline & Device 1 & Device 2 & Device 3 \\
\hline Designation & eCareCompanion & Genesis Telemonitor & Honeywell HomMed \\
\hline Portability & Yes & Yes & Yes/no \\
\hline Invasiveness & Yes & Yes & Yes \\
\hline Interface & Custom and simple & Custom and simple & Custom and simple \\
\hline Connectivity & $\begin{array}{l}\text { Wi-Fi } \\
\text { Bluetooth }\end{array}$ & $\begin{array}{l}\text { Wi-Fi } \\
\text { Bluetooth } \\
\text { 3G/4G }\end{array}$ & $\begin{array}{l}\text { Direct connection to } \\
\text { device } \\
\text { Wi-Fi }\end{array}$ \\
\hline Interoperability & No & No & No \\
\hline
\end{tabular}

Table 5 Comparison of technical specifications of telemonitoring systems used in Heart Failure

\begin{tabular}{l|l|l|l}
\hline & Device 1 & Device 2 & Device 3 \\
\hline Designation & VITALS360 & TCare cardio monitor & Smartheart \\
\hline Portability & Yes & Yes & Yes \\
\hline Invasiveness & No & No & No \\
\hline Interface & Custom and simple & Custom and simple & Custom and simple \\
\hline Connectivity & Wi-Fi & Bluetooth GSM/HDSPA & BluetoothWi-Fi \\
\hline Interoperability & No & No & No \\
\hline
\end{tabular}

million people living with the disease in the world $[35,36]$. Due to the progression of the disease, the decompensation is frequent, which leads to hospitalizations also more frequent [37]. In the USA alone, the number of hospitalizations exceeds one million per year, which significantly increases the costs associated with the disease for both the patient and the health system [35]. According to Liu et al. [38, 39], costs directly and indirectly involving the treatment of heart failure in the USA were around $\$ 39.2$ billion in 2010 , with only re-hospitalizations accounting for approximately $\$ 31$ billion of dollars spent.

The devices described are VITALS360 ${ }^{\circledR}$, TCare Cardio Monitor and Smartheart $^{\mathrm{TM}}$. All devices and systems are portable. Obtaining the ECG tracing is non-invasive and is the main test performed with these devices and telemonitoring systems. In VITALS $360^{\circledR}$, the ECG is performed directly on the device. In the remaining devices, the ECG trace is sent via Bluetooth from external ECG devices. Results are sent through Wi-Fi in the case of TCare Cardio Monitor through GSM/HDSPA. The patient-to-patient interface is simple and customizable to the patient on all devices [12, 40, 41].

Table 5 reviews the technical specifications of the devices and telemonitoring systems found for Heart Failure. Following the path of the telemonitoring devices for the previous pathologies, the interoperability between devices and systems of different brands is inexistent. 


\section{Proposal for Future Telemonitoring IoT System}

The proposed trend-based telemonitoring system is shown in Fig. 1. This is composed of four components (1) wearable monitoring device; (2) smartphone (health application); (3) cloud; (4) call centre, patient and doctor.

The proposal considers a wearable and non-invasive device that is placed in contact with the patient's skin, acquires the indicated biological and physical parameters for the disease in question. The proposed non-invasive wearable monitoring device consists of one or more biological and physical parameter measurement sensors, a microcontroller, a battery and a Bluetooth Low Energy (BLE) integrated circuit. The data is collected by the monitoring device and sent to the smartphone via BLE.

In the smartphone there is a health application that collects and filters firstly the data, according to an algorithm that limits the values. These limits should be adjusted and personalized for each patient, being only sent to the cloud values higher than the reference values. Inter-operation for the remote cloud-based system could be helped by using common health data APIs/wellness data APIs (some of which are already on the market) or health data description taxonomies (currently a focus of research). In the cloud there is another algorithm that decides according to the results received: (1) it communicates some message to the patient. If this condition occurs, an information report is also sent to the specialist doctor who follows the patient. (2) If it is sent to the call centre, the results are outside the limit range and the medical specialists will decide what procedures to take, contact the patient immediately giving instructions of procedures to take, contact the emergency service to move to the patient or even contact the patient's personal doctor to help make a decision.

This ideal IoT telemonitoring system will be able to interoperate with programs developed by different software houses, in order to allow the flow of information of patients' clinical data between healthcare systems at different levels, ensuring the confidentiality and security of data.

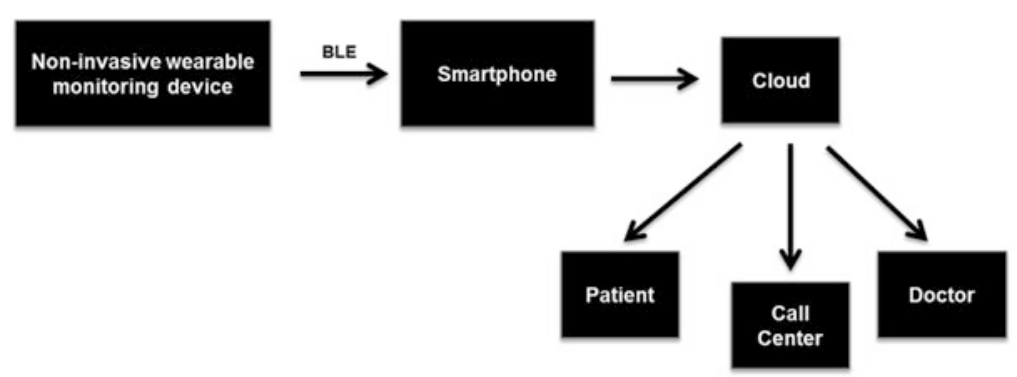

Fig. 1 Scheme of the proposal for future IoT telemonitoring system 


\section{Conclusions}

Telemonitoring for chronic patients can bring great benefits to both the patient and health systems. For the patient the increase of care in the self-management of the disease consequently leads to an improvement in the health condition and quality of life, for the health systems the distribution of resources is achieved in an equitable way reaching the majority of possible chronic population.

The enlargement of this practice applied to chronic pathologies will be a reality in the near future, not forgetting that until this premise becomes real some gaps will have to be overcome as the creation of interoperability programs between health support systems, which is the main failure of the devices and systems telemonitoring existing on the market.

\section{References}

1. World Health Organization: Noncommunicable diseases. http://www.who.int/topics/ noncommunicable_diseases/en/. Accessed 04 Feb 2017

2. Ho, K.: Supporting heart failure patient transitions from acute to community care with home telemonitoring technology: a protocol for a provincial randomized controlled trial (TEC4Home). JMIR Res. Protoc. 5(4), e198 (2016)

3. Saner, H., Van Der Velde, E.: eHealth in cardiovascular medicine: a clinical update. Eur. J. Prev. Cardiol. 23, 5-12 (2016)

4. Paré, G., Moqadem, K., Pineau, G., St-Hilaire, C.: Clinical effects of home telemonitoring in the context of diabetes, asthma, heart failure and hypertension: a systematic review. J. Med. Internet Res. 12(2), e21 (2010)

5. Esteban, C., Moraza, J., Iriberri, M., Aguirre, U., Goiria, B., Quintana, J.M., Aburto, M., Capelastegui, A.: Outcomes of a telemonitoring-based program (telEPOC) in frequently hospitalized COPD patients. Int. J. COPD. 11, 2919-2930 (2016)

6. Bashshur, R.L., Shannon, G.W., Smith, B.R., Alverson, D.C., Antoniotti, N., et al.: The empirical foundations of telemedicine interventions for chronic disease management. Telemed. J. E Health. 20, 769-800 (2014)

7. Gyllensten, I.C., Crundall-Goode, A., Aarts, R.M., Goode, K.M.: Simulated case management of home telemonitoring to assess the impact of different alert algorithms on work-load and clinical decisions. BMC Med. Inform. Decis. Mak. 17(1), 11 (2017)

8. Craig, M.E., Hattersley, A., Donaghue, K.C.: Definition, epidemiology and classification of diabetes in children and adolescents. Pediatr. Diabetes. 15, 4-17 (2014)

9. Cariou, B., Eddy, D., Schwarz, P., Gumprecht, J., Smith, U., et al.: The diabetes epidemic and its impact on Europe. In: European Diabetes Leadership Forum, p. 44. OECD, Paris (2012)

10. Wild, S.H., Hanley, J., Lewis, S.C., McKnight, J.A., McCloughan, L.B., et al.: Supported telemonitoring and glycemic control in people with type 2 diabetes: the telescot diabetes pragmatic multicenter randomized controlled trial. PLoS Med. 13, 1-16 (2016)

11. Edevice: HealthGO Mini. http://www.edevice.com/products/healthgo-mini. Accessed 08 Jun 2017

12. VoCare: Vitals $360^{\circledR}$. http://vocare.com/vitals360/. Accessed 06 Jun 2017

13. TelehealthSolutions: CareHomePod. https://www.thsl.co.uk/products/carehomepod. Accessed 08 Jun 2017

14. VoCare ${ }^{\circledR}$. Vitals $360^{\circledR}$ user manual. p. 13 (2013) 
15. Pedone, C., Lelli, D.: Systematic review of telemonitoring in copd: an update. Pneumonol. Alergol. Pol. 83, 476-484 (2015)

16. Vivekanandan, S., Devanand, M.: Remote monitoring for diabetes disorder: pilot study using InDiaTel prototype. Eur. Res. Telemed. 4, 63-69 (2015)

17. Fitzsimmons, D.A., Thompson, J., Bentley, C.L., Mountain, G.A.: Comparison of patient perceptions of Telehealth-supported and specialist nursing interventions for early stage COPD: a qualitative study. BMC Health Serv. Res. 16, 420 (2016)

18. Merone, M., Pedone, C., Capasso, G., Incalzi, R.A., Soda, P.: A decision support system for tele-monitoring COPD-related worrisome events. IEEE J. Biomed. Health Informatics. 2194, 2168-2194 (2017)

19. Mulpuru, S., Mckay, J., Ronksley, P.E., Thavorn, K., Kobewka, D.M., Forster, A.J.: Factors contributing to high-cost hospital care for patients with COPD. Int. J. COPD. 12, 989-995 (2017)

20. Fonseca, J.A., Costa-Pereira, A., Delgado, L., Silva, L.N., Magalhães, M., Castel-Branco, M.G., Vaz, M.: Pulmonary function electronic monitoring devices: a randomized agreement study. Chest J. 128, 1258-1265 (2005)

21. Masimo: $\mathrm{iSpO} 2^{\circledR}$ pulse oximeter. http://masimopersonalhealth.com/products/ispo2-pulseoximeter/. Accessed 07 Jun 2017

22. Masimo Shop. http://masimopersonalhealth.com/shop/. Accessed 07 Jun 2017

23. Wilson, C., Campbell, S.M., Luker, K.A., Caress, A.L.: Referral and management options for patients with chronic kidney disease: perspectives of patients, generalists and specialists. Health Expect. 18, 325-334 (2015)

24. Cuevas, J.R., Dominguez, E.L., Velazquez, Y.H.: Telemonitoring system for patients with chronic kidney disease undergoing peritoneal dialysis. IEEE Lat. Am. Trans. 14, 2000-2006 (2016)

25. Raman, M., Green, D., Middleton, R.J., Kalra, P.A.: Older people with chronic kidney disease: definition, and influence of biomarkers and medications upon cardiovascular and renal outcomes. J. Ren. Care. 42, 150-161 (2016)

26. Thilly, N., Chanliau, J., Frimat, L., Combe, C., Merville, P., et al.: Cost-effectiveness of home telemonitoring in chronic kidney disease patients at different stages by a pragmatic randomized controlled trial (eNephro): rationale and study design. BMC Nephrol. 18, 126 (2017)

27. Singh, P., Ricksten, S.E., Bragadottir, G., Redfors, B., Nordquist, L.: Renal oxygenation and haemodynamics in acute kidney injury and chronic kidney disease. Clin. Exp. Pharmacol. Physiol. 40, 138-147 (2013)

28. Trifirò, G., Fatuzzo, P.M., Ientile, V., Pizzimenti, V., Ferrajolo, C., et al.: Expert opinion of nephrologists about the effectiveness of low-protein diet in different stages of chronic kidney disease (CKD). Int. J. Food Sci. Nutr. 65, 1027-1032 (2014)

29. eQOL: Our solutions empower patients to manage their care outside of the hospital. https:// eqol.ca/index.php (2017). Accessed 11 Jun 2017

30. Jeffs, L., Jain, A.K., Man, R.H., Onabajo, N., Desveaux, L., et al.: Exploring the utility and scalability of a telehomecare intervention for patients with chronic kidney disease undergoing peritoneal dialysis - a study protocol. BMC Nephrol. 18, 155 (2017)

31. Kenne, E., Nickel, K.F., Long, A.T., Fuchs, T.A., Stavrou, E.X., et al.: Factor XII: a novel target for safe prevention of thrombosis and inflammation. J. Intern. Med. 278, 571-585 (2015)

32. Araújo, A.C.O., Domingues, R.B., van Bellen, B.: Determinação do INR: Comparação entre método convencional e dispositivo portátil. J. Vasc. Bras. 13, 88-93 (2014)

33. Philips: eCareCompanion patient app. http://www.usa.philips.com/healthcare/product/ HCNOCTN483/ecarecompanion-patient-app-your-patients-gateway-to-care. Accessed 09 Jun 2017

34. Honeywell Life Care: Genesis telemonitor - targeted solution. https:// www.honeywelllifecare.com/lifestream-products/legacy-products-and-software/genesistelemonitor/. Accessed 09 Jun 2017

35. Pyramidhhs. http://pyramidhhs.com/index.php/services/home-health/missouri-telemonitoringremote-disease-management. Accessed 09 Jun 2017 
36. Pyramid HHS. http://pyramidhhs.com/index.php/contact-us. Accessed 09 Jun 2017

37. Tadic, M., Pieske-Kraigher, E., Cuspidi, C., Genger, M., Morris, D.A., et al.: Left ventricular strain and twisting in heart failure with preserved ejection fraction: an updated review. Heart Fail. Rev. 22, 371-379 (2017)

38. Knox, L., Rahman, R.J., Beedie, C.: Quality of life in patients receiving telemedicine enhanced chronic heart failure disease management: a meta-analysis. J. Telemed. Telecare. 23(7), 639649 (2017)

39. Pazos-López, P., Peteiro-Vázquez, J., Carcía-Campos, A., García-Bueno, L., de Torres, J.P.A., Castro-Beiras, A.: The causes, consequences, and treatment of left or right heart failure. Vasc. Health Risk Manag. 7, 237-254 (2011)

40. TCare Cardio Monitor. http://www.tcare.pt/servicos/tcare-cardio-monitor/. Accessed 04 Jun 2017

41. SHL Telemedicine: SmartHeart. http://www.shl-telemedicine.com/portfolio/smartheart/. Accessed 08 Jun 2017 